\title{
Exercise protocol induces muscle, tendon, and bone adaptations in the rat shoulder
}

\author{
Sarah Ilkhanipour Rooney ${ }^{1}$ \\ Emanuele Loro ${ }^{2}$ \\ Joseph J. Sarver ${ }^{3}$ \\ Cathryn D. Peltz ${ }^{4}$ \\ Michael W. Hast ${ }^{1}$ \\ Wei-Ju Tseng ${ }^{1}$ \\ Andrew F. Kuntz ${ }^{1}$ \\ X. Sherry Liu ${ }^{1}$ \\ Tejvir S. Khurana ${ }^{2}$ \\ Louis J. Soslowsky ${ }^{1}$
}

1 McKay Orthopaedic Research Lab, University of Pennsylvania, Philadelphia, PA, USA

2 Department of Physiology, Pennsylvania Muscle Institute, University of Pennsylvania School of Medicine, Philadelphia, PA, USA

3 Biomedical Engineering, Drexel University, Philadelphia, PA, USA; McKay Orthopaedic Research Lab, University of Pennsylvania, Philadelphia, PA, USA

4 Bone \& Joint Center, Henry Ford Hospital, Detroit, MI, USA; McKay Orthopaedic Research Lab, University of Pennsylvania, Philadelphia, PA, USA

Corresponding author:

Louis J. Soslowsky

McKay Orthopaedic Research Lab, University of Pennsylvania

424 Stemmler Hall

36th St. and Hamilton Walk

19104 Philadelphia, USA

E-mail: soslowsk@upenn.edu

\section{Summary}

Background: a rat model of supraspinatus overuse has suggested mechanisms governing tendon degeneration; however, delineating which changes are pathologic or simply physiologic adaptations to increased loading remains a question. The objective of this study was to develop and characterize a rat exercise model that induces systemic and local shoulder adaptations without mechanical injury to the supraspinatus tendon.

Methods: exercise rats completed a treadmill training protocol for 12 weeks. Body, fat pad, and heart weights were determined. Supraspinatus tendon collagen content, cross-sectional area, and mechanical properties were measured. Supraspinatus muscle cross-sectional area, weight, and the expression of mitochondrial oxidative phosphorylation (OXPHOS) proteins were measured. Humeri were analyzed with $\mu \mathrm{CT}$ and mechanically tested.

Results: exercise decreased fat pad mass. Supraspinatus muscle hypertrophied and had increased OXPHOS proteins. Humerus trabecular bone had increased anisotropic orientation, and cortical bone showed increased bone and tissue mineral density. Importantly, the supraspinatus tendon did not have diminished mechanical properties, indicating that this protocol was not injurious to the tendon.

Conclusion: this study establishes the first rat exercise protocol that induces adaptations in the shoulder. Future research can use this as a comparison model to study how the supraspinatus tendon adapts to loading and undergoes degeneration with overuse.

KEY WORDS: mechanics, rotator cuff, supraspinatus, tendinopathy.

\section{Introduction}

Rotator cuff tendinopathy, which primarily affects the supraspinatus tendon, is a frequent clinical condition. This tendon degeneration accounts for $29 \%$ of reported shoulder complaints ${ }^{1}$ and is common in laborers with jobs requiring repetitive overhead work and athletes. Supraspinatus tendinopathy in elite swimmers correlated with the hours and kilometers swum per week, highlighting the role of overuse in the development of the condition ${ }^{2}$. While late-stage tendinopathy has been fairly well characterized, earlier stages of tendon degeneration have not, partially due to the lack of an appropriate comparison system. Early stage tendinopathy is challenging to study because patients do not seek invasive treatment prior to or at the onset of symptoms, and defining the onset of tendon pathology is difficult. For these reasons, animal models, which allow for controlled, repeatable injuries, are vital to learning about the pathogenesis of tendon degeneration.

A rat model of supraspinatus overuse ${ }^{3}$ has suggested mechanisms governing tendon degeneration; however, delineating which changes are pathologic or sim- 
ply physiologic adaptations to increased loading remains a question. The development of a non-injurious exercise model to which the rat model of supraspinatus overuse can be compared is critical to the advancement of tendinopathy research; this model has not been created previously. Such a model should mimic the key adaptive properties of the shoulder to exercise without injuring the supraspinatus tendon.

The objective of this study was to develop and characterize a rat exercise model that induces systemic and local shoulder adaptations without mechanical injury to the supraspinatus tendon. We hypothesized that a mild treadmill training protocol would produce adaptations consistent with exercise in the supraspinatus tendon and muscle and the humerus. Specifically, aerobic exercise is often associated with decreased body and fat mass and increased capacity for aerobic metabolism within muscle. Highly aerobic activity can cause heart hypertrophy. Resistance exercise can produce muscle hypertrophy and increased bone properties, such as density. Damaged, pathologic tendon has inferior elastic mechanical properties ${ }^{3}, 4$; therefore, we sought to confirm that this beneficial exercise protocol does not compromise the supraspinatus tendon's mechanical integrity.

\section{Materials and methods}

\section{Study design and treadmill protocol}

This study was conducted in accordance with the ethical standards set by Padulo et $\mathrm{al}^{5}$. Sixteen adult, male Sprague-Dawley rats were ordered by weight (400-450 g) and distributed evenly between exercise (EX; $n=8)$ and control cage activity $(C A ; n=8)$ groups following approval from the university's Institutional Animal Care and Use Committee. To account for potential body weight loading effects on musculoskeletal tissues, animals were ordered within a small weight range (rather than age) that corresponds to $\sim 15$ weeks of age ${ }^{6}$. Exercise animals underwent two weeks of treadmill training and then walked on a flat treadmill at a constant speed of 10 meters/minute for 1 hour per day during normal work hours (between 8:00 am and 5:00 pm), 5 days per week, for 12 weeks $^{7}$. The treadmill (Nordic Track C2155, Logan, UT, USA) was custom-geared to allow for slower speeds. The treadmill was kept flat and the speed was checked with a digital handheld tachometer (HT-346, Ono Sokki, Japan) during every exercise session. At $10 \mathrm{~m} / \mathrm{min}$, the animals are walking at only $10-15 \%$ of their galloping speed 8,9 . Control animals maintained normal cage activity for the duration of the study. The rats were housed in an AALAC accredited facility that maintained a 12/12 hour light/dark cycle, temperatures between $20-26^{\circ} \mathrm{C}$, and humidity between $30-70 \%$, as described in the Guide for Care and Use of Laboratory Animals ${ }^{10}$. Rats were euthanized, weighed, and stored at $-20^{\circ} \mathrm{C}$. Percent body weight change was calculated between the initial (arrival) and final (sacrifice) time points.

\section{Tissue harvest}

Rats were thawed and the right supraspinatus muscle and tendon were dissected free from the humerus and weighed using a digital scale (Ohaus Corp., Pine Brook, NJ, USA) with $0.01 \mathrm{~g}$ readability. Tendon at the bony insertion site was isolated from the muscle for o-Hydroxy-proline (OHP) assay. Muscle crosssectional area (CSA) was measured with a custom laser device accurate to $0.05 \mathrm{~mm}^{2}(200 \mathrm{~Hz}$ sampling rate $)^{11}$. The superficial region of the supraspinatus muscle ${ }^{12}$ was collected for protein analysis. The heart and retroperitoneal and epididymal fat pads were dissected and weighed.

\section{Tendon mechanics}

Rats were thawed, and the left supraspinatus tendon and humerus were dissected and prepared for tensile, elastic mechanical testing, as previously described $^{13}$. Briefly, tendons were finely dissected to remove muscle and excess connective tissue, and Verhoeff's stain lines for optical strain measurements were applied at the bony insertion site and 2, 4 , and $8 \mathrm{~mm}$ proximally. Tendon cross-sectional area was measured with a custom laser device ${ }^{11}$. Cyanoacrylate was used to fix the tendon between two pieces of sandpaper at the $8 \mathrm{~mm}$ stain line, and the sandpaper was subsequently gripped with an aluminum alloy custom screw clamp. Humeri were potted in polymethyl methacrylate, and a custom fixture secured the potted humerus for testing with a universal testing machine (Instron, Norwood MA, USA) affixed with a $100 \mathrm{~N}$ load cell (accurate to $0.25 \%$ of the reading). Tendons were submerged in a $39^{\circ} \mathrm{C}$ phosphate-buffered saline solution bath and underwent preconditioning (preload to $0.1 \mathrm{~N}, 10 \mathrm{cy}$ cles from 0.1 to $0.5 \mathrm{~N}$ at $1 \% / \mathrm{s}$, hold at $0.1 \mathrm{~N}$ for 300 $\mathrm{s}$, hold at $5 \%$ strain for $600 \mathrm{~s}$, hold at $0.1 \mathrm{~N}$ for $60 \mathrm{~s}$ ) prior to a ramp to failure at an applied rate of $0.3 \% / \mathrm{s}$. Local 2D Lagrangian strain of the tendon during the ramp to failure was measured by tracking the stain lines using a custom texture-tracking program (MATLAB, Natick, MA, USA). Elastic modulus was calculated as the slope of the linear region of the stress-strain curve.

\section{Tendon collagen}

$\mathrm{OHP}$, a measure of total collagen content, was determined for supraspinatus tendon samples as previously described ${ }^{14,} 15$ and normalized to tendon wet weight. Briefly, tendons were dried and digested in proteinase $\mathrm{K}$ solution. Hydrolysis with $\mathrm{HCl}$ was performed in heated glass vials. Samples were lyophilized and re-suspended in water. A colorimetric reaction was used to determine the OHP content, which was converted to total collagen using a 1:14 ratio of $\mathrm{OHP}$ to collagen. 


\section{Muscle protein}

A western blot was performed to quantify supraspinatus muscle mitochondrial proteins. Supraspinatus muscle superficial region samples were homogenized with RIPA buffer plus EDTA-free protease inhibitor (Roche Diagnostics, Indianapolis, IN, USA) and phosphatase inhibitor (Roche Diagnostics, Indianapolis, IN, USA). Tissue homogenates were centrifuged for 10 minutes at $4^{\circ} \mathrm{C}(12,000 \times \mathrm{g})$. Total protein was quantified using a BCA protein assay kit (Pierce, Thermo Scientific, Rockford, IL, USA). From each sample, 20 $\mu \mathrm{g}$ of total protein was loaded into a well of a $4-15 \%$ gradient TGX precast SDS-PAGE gel (Bio-Rad, Hercules, CA, USA) and electrophoresed. Proteins were blotted into a PVDF membrane (Bio-Rad, Hercules, CA, USA), blocked for non-specific binding sites, and probed for oxidative phosphorylation (OXPHOS) complexes I-V using Total OXPHOS antibody cocktail (MitoSciences, Eugene, OR, USA). After incubation with a specific HRP-conjugated antibody, bands were visualized by chemiluminescence (C-DiGit, LI-COR, Lincoln, NE, USA). Integrated optical density of each band was calculated with commercial software. Membranes were stripped and re-probed with an antibody against $\alpha$-tubulin for normalization. The relative quantity of normalized target protein in the exercise group compared to cage activity was calculated.

\section{Bone micro-Computed Tomography $(\mu \mathrm{CT})$}

MCT (VivaCT40, ScancoMedical AG, Brüttisellen, Switzerland) was used to determine trabecular and cortical humerus bone structure. For trabecular bone, a $2 \mathrm{~mm}$ region just distal to the humeral head growth plate was scanned (15 $\mu \mathrm{m}$ isotropic voxels), followed by a semi-automatic segmentation of trabecular bone compartment from cortex. For cortical bone, a $1.5 \mathrm{~mm}$ region at $60 \%$ of the humerus length was scanned (35 $\mu \mathrm{m}$ isotropic voxels). Then, bone tissue was thresholded from marrow using a global thresholding technique and subjected to standard microstructural analysis with Scanco software ${ }^{16}$. Upon blinded visual inspection of all samples, a threshold value of 849 $\mathrm{mgHA} / \mathrm{ccm}$ was chosen for trabecular analysis, and $979 \mathrm{mgHA} / \mathrm{ccm}$ was chosen for cortical analysis. Both cortical and trabecular bone mineral density (BMD) were quantified as the total bone content divided by the bulk volume. Cortical and trabecular bone tissue mineral density (TMD) were calculated as the total bone content divided by the bone volume. The parameters that evaluate the quantity of trabecular bone, including trabecular bone volume, number, thickness, and spacing were derived. Degree of anisotropy reflects the orientation of the trabecular bone network. Connectivity density quantifies trabecular connectivity by calculating the number of handles or closed loops in a trabecular network. Structure model index (SMI) estimates, on average, the plate versus rod characteristics of trabecular bone, ranging from 0 for an ideal plate structure to 3 for an ideal rod structure. Second moment of area (I) of the humeral shaft was calculated from custom software analysis of $\mu \mathrm{CT}$ images.

\section{Bone 3-point bending}

Following $\mu \mathrm{CT}$ scanning, symmetric 3-point bending was performed to determine mechanical properties of humerus cortical bone. Humeri were dissected free of all soft tissue. A custom fixture attached to a universal testing machine (Instron, Norwood MA, USA) created a 3-point bend in the humeral shaft until fracture using a protocol adapted from Raab et al. ${ }^{17}$. Briefly, the humeri were centered on two $3.175 \mathrm{~mm}$ diameter pins spaced $11 \mathrm{~mm}$ apart with the deltoid tuberosity pointing upwards. A similar pin was affixed to the crosshead, which moved at a constant velocity of 5 $\mathrm{mm} / \mathrm{min}$ until fracture occurred. The bones were kept hydrated throughout the course of testing by spraying them with phosphate-buffered saline solution. Bending modulus ( $E$ ) was calculated as $\mathrm{FL}^{3} / 48 \delta \mathrm{I}$, where $\mathrm{F}$ is the failure load, $L$ is the distance between the two base pins $(11 \mathrm{~mm}), \delta$ is the deflection, and $I$ is the second moment of area. Flexural rigidity was calculated as El. The max flexural stress was calculated as $(F / 2)^{\star}(\mathrm{L} / 2)^{\star} \gamma / \mathrm{l}$, where $\gamma$ is the perpendicular distance to the neutral axis determined from $\mu \mathrm{CT}$ images.

\section{Statistics}

Comparisons between exercise and cage activity groups were made with 1-tailed t-tests. Significance was set at $p \leq 0.05$ and trends at $p \leq 0.1$. At a power of $80 \%$ and the given sample size of 8 specimens per group, we can use a one-tailed t-test to measure a significant difference $(\alpha=0.05)$ of an effect size Cohen's $\delta$ equal to 1.3 .

\section{Results}

Exercise animals trended toward reduced body weight gain over the course of the study $(p=0.07)$ and had significantly decreased retroperitoneal (-45\%, $p=0.00004)$ and epididymal $(-39 \%, p=0.0001)$ fat pad masses (Fig. 1). An $8 \%$ increase in supraspinatus muscle mass was measured ( $p=0.008)$, and no change was detected in heart mass (Fig. 1). Supraspinatus muscle cross-sectional area significantly increased $10 \%$ with exercise $(p=0.008)($ Tab. 1$)$.

\section{Tendon mechanics}

Supraspinatus tendon cross-sectional area and elastic modulus were not different between groups (Tab. 1). Failure load significantly increased with exercise $(p=0.04)$, and failure stress trended toward increased $(p=0.09)$ (Tab. 1). 


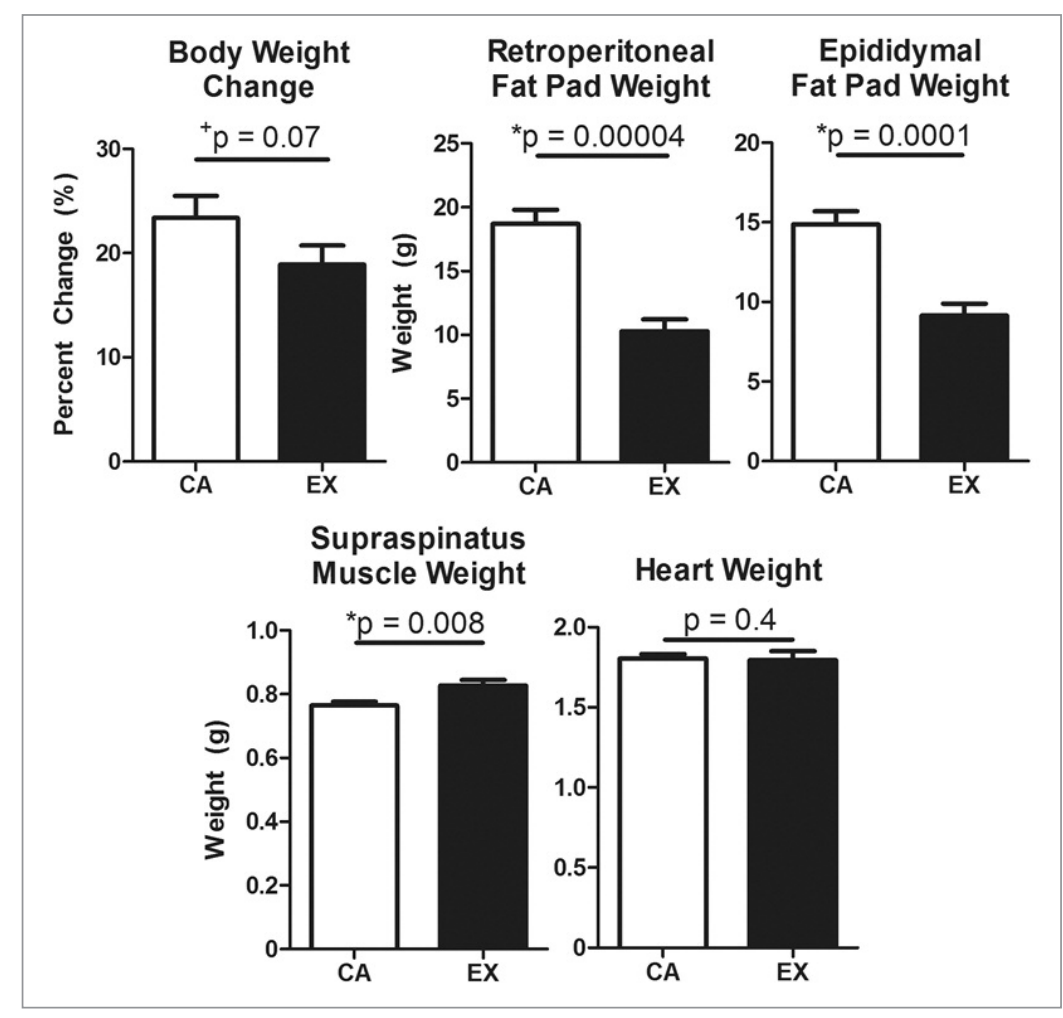

Figure 1. Consistent with exercise, treadmill-trained rats trended toward decreased body weight over time and had significantly decreased fat pad weight, increased supraspinatus muscle weight, and no change in heart weight. Mean + SEM. \% Coefficient of Variation: body weight change $=26 \% \mathrm{CA}, 27 \% \mathrm{EX}$; retroperitoneal $=15 \% \mathrm{CA}, 26 \% \mathrm{EX}$; epdidymal $=13 \%$ CA, 24\% EX; muscle $=3 \% \mathrm{CA}, 6 \% \mathrm{EX}$; heart $=4 \%$ CA, $9 \%$ EX.

Table 1. Shoulder adaptations to exercise protocol. Treadmill-trained rats showed adaptations consistent with exercise in supraspinatus tendon and muscle, and trabecular ( $\mathrm{Tb}$ ) and cortical (Ct) humerus bone.

\begin{tabular}{|c|c|c|c|c|c|c|c|c|}
\hline \multirow[t]{2}{*}{ Tissue } & \multirow[t]{2}{*}{ Measurement } & \multicolumn{3}{|c|}{ CA } & \multicolumn{3}{|c|}{ EX } & \multirow[t]{2}{*}{ p-value } \\
\hline & & Mean & SD & $\% \mathrm{CV}$ & Mean & SD & $\% \mathrm{CV}$ & \\
\hline Muscle & $\mathrm{CSA}\left(\mathrm{mm}^{2}\right)$ & 34.3 & 3.9 & 11 & 37.7 & 2.6 & 7 & *0.04 \\
\hline \multirow[t]{5}{*}{ Tendon } & $\mathrm{CSA}\left(\mathrm{mm}^{2}\right)$ & 1.97 & 0.42 & 21 & 1.97 & 0.28 & 14 & 0.5 \\
\hline & Failure Load (N) & 22.3 & 5.8 & 26 & 26.8 & 3.0 & 11 & *0.04 \\
\hline & Failure Stress (MPa) & 11.6 & 3.4 & 29 & 14.0 & 3.2 & 23 & +0.09 \\
\hline & Modulus (MPa) & 100 & 42 & 42 & 101 & 24 & 24 & 0.5 \\
\hline & Collagen/Wet Weight (\%) & 25 & 5 & 20 & 28 & 1 & 5 & +0.07 \\
\hline \multirow[t]{7}{*}{ Bone } & Tb Connective Density $\left(1 / \mathrm{mm}^{3}\right)$ & 60 & 12 & 20 & 70 & 12 & 17 & +0.07 \\
\hline & Tb Number $(1 / \mathrm{mm})$ & 3.1 & 0.7 & 21 & 3.5 & 0.4 & 11 & +0.10 \\
\hline & Tb Spacing $(\mathrm{mm})$ & 0.34 & 0.09 & 27 & 0.28 & 0.04 & 14 & +0.08 \\
\hline & Tb Degree of Anisotropy & 1.79 & 0.07 & 4 & 1.86 & 0.07 & 4 & ${ }^{*} 0.03$ \\
\hline & Ct Thickness (mm) & 0.77 & 0.02 & 3 & 0.80 & 0.03 & 4 & $* 0.05$ \\
\hline & $\mathrm{Ct} \mathrm{BMD}\left(\mathrm{mgHA} / \mathrm{cm}^{3}\right)$ & 1116 & 12 & 1 & 1126 & 10 & 0.9 & *0.05 \\
\hline & Ct TMD (mgHA/cm³) & 1212 & 18 & 1 & 1227 & 7 & 0.6 & *0.02 \\
\hline
\end{tabular}

$\mathrm{SD}=$ standard deviation, $\% \mathrm{CV}=\%$ coefficient of variation, ${ }^{*}=$ significant, ${ }^{+}=$trend

\section{Tendon collagen}

Collagen content of the supraspinatus tendon normalized to wet weight trended toward increased in the exercise group $(p=0.07)($ Tab. 1$)$.

\section{Muscle protein}

The superficial region of the supraspinatus muscle had increased oxidative phosphorylation proteins
(Fig. 2). These proteins included significantly increased presence of OXPHOS complexes I-III, and trends toward increased presence of OXPHOS complexes IV and V.

\section{Bone $\mu \mathrm{CT}$}

Trabecular bone in the exercise group demonstrated trends toward increased trabecular number $(p=0.1)$ and connectivity $(p=0.07)$ and decreased spacing ( $p$ 
$=0.08)$. Trabecular bone had significantly greater degree of anisotropy in the exercise group $(p=0.03)$ (Tab. 1). No differences were found in trabecular bone volume, SMI, thickness, bone mineral density (BMD), or tissue mineral density (TMD) (Tab. 2).

Cortical bone in the exercise group had significantly increased thickness, BMD, and TMD (Tab. 1) with no changes in volume or second moment of area (Tab. 2).

\section{Bone 3-point bending}

No differences were found for max load, modulus, flexural rigidity, or max stress (Tab. 2).

\section{Discussion}

After 12 weeks of a mild treadmill protocol, rats showed systemic and local shoulder changes consistent with exercise. The animals had reduced fat pad mass and trended toward reduced body weight gain. Heart mass did not change, indicating that this protocol does not tax the cardiovascular system. Locally, the rat shoulder showed adaptations to this exercise protocol. As a result of treadmill walking, the supraspinatus muscle hypertrophied (increased crosssectional area and mass), consistent with a response to increased loading, and had increased oxidative phosphorylation proteins, consistent with a response

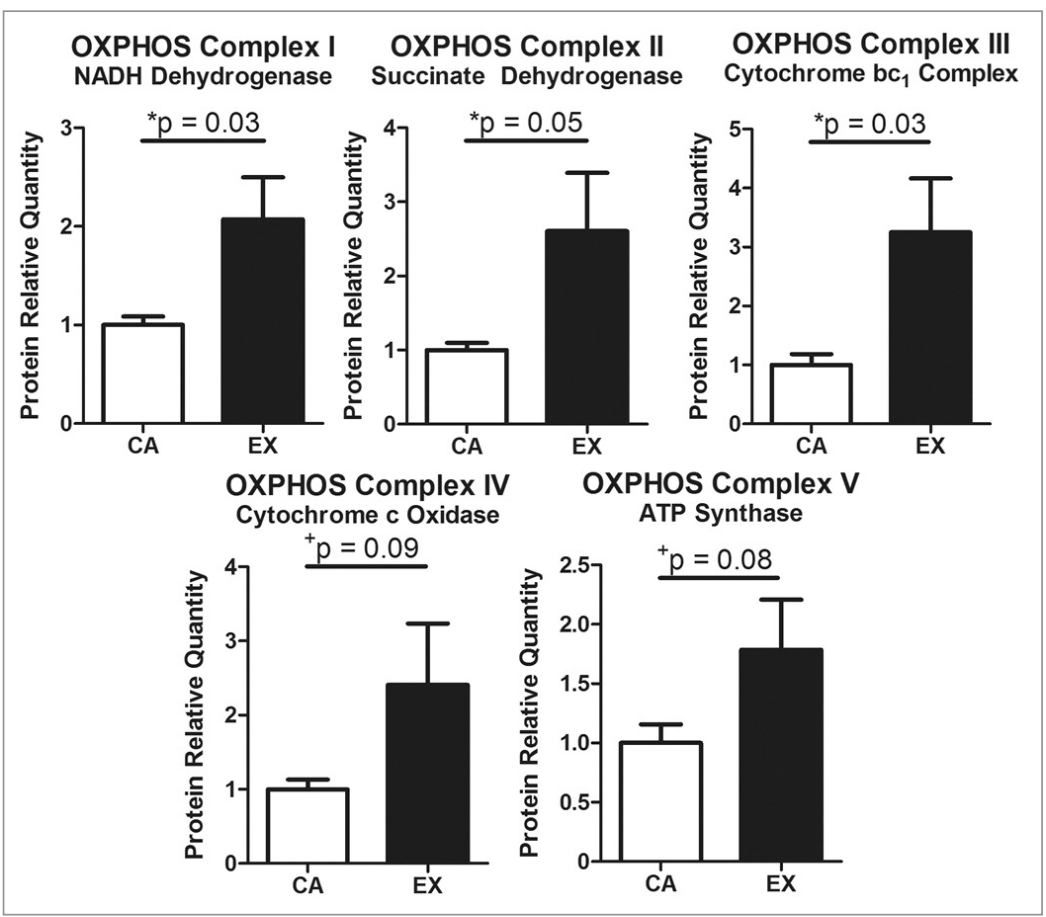

Figure 2. Consistent with adaptations to aerobic exercise, treadmill-trained rats had increased expression of oxidative phosphorylation proteins. Relative quantity of exercise to cage activity after normalizing by tubulin. Mean + SEM. \% Coefficient of Variation: Complex I $=21 \%$ CA, $58 \%$ EX; Complex II = 24\% CA, $85 \%$ EX; Complex III $=46 \%$ CA, $80 \%$ EX; Complex IV $=32 \%$ CA, $97 \%$ EX; Complex V $=38 \%$ CA, $67 \%$ EX.

Table 2. Humerus Bone Measurements Unchanged with Exercise. Insignificant results of $\mu \mathrm{CT}$ and 3-point bend testing of trabecular (Tb) and cortical (Ct) humerus bone.

\begin{tabular}{|c|c|c|c|c|c|c|c|c|}
\hline \multirow[t]{2}{*}{ Assay } & \multirow[t]{2}{*}{ Measurement } & \multicolumn{3}{|c|}{ CA } & \multicolumn{3}{|c|}{ EX } & \multirow[t]{2}{*}{ p-value } \\
\hline & & Mean & SD & $\% \mathrm{CV}$ & Mean & SD & $\% \mathrm{CV}$ & \\
\hline \multirow[t]{7}{*}{$\mu \mathrm{CT}$} & Tb Bone Volume $\left(\mathrm{mm}^{3}\right)$ & 5.45 & 1.08 & 20 & 6.02 & 1.08 & 18 & 0.2 \\
\hline & Tb Structure Model Index & 1.52 & 0.40 & 26 & 1.57 & 0.12 & 8 & 0.4 \\
\hline & Tb Thickness (mm) & 0.09 & 0.01 & 8 & 0.09 & 0.01 & 7 & 0.4 \\
\hline & $\mathrm{Tb}$ BMD (mgHA/cm³) & 308 & 48 & 16 & 325 & 30 & 9 & 0.2 \\
\hline & $\mathrm{Tb}$ TMD $\left(\mathrm{mgHA} / \mathrm{cm}^{3}\right)$ & 875 & 7 & 0.8 & 871 & 11 & 1 & 0.2 \\
\hline & Ct Bone Volume $\left(\mathrm{mm}^{3}\right)$ & 8.86 & 1.06 & 12 & 8.70 & 0.49 & 6 & 0.4 \\
\hline & Second Moment of Area $\left(\mathrm{mm}^{4}\right)$ & 5.0 & 1.3 & 27 & 4.7 & 1.0 & 21 & 0.3 \\
\hline 3-Point & Max Load (N) & 255 & 43 & 17 & 238 & 18 & 8 & 0.2 \\
\hline Bending of & Max Stress (MPa) & 224 & 18 & 8 & 224 & 32 & 14 & 0.5 \\
\hline Humeral & Bending Modulus (GPa) & 2.05 & 0.52 & 25 & 2.19 & 0.52 & 24 & 0.3 \\
\hline Shaft & Flexural Rigidity (N-mm²) & 9700 & 530 & 5 & 9860 & 790 & 8 & 0.3 \\
\hline
\end{tabular}

$\overline{\mathrm{SD}}=$ standard deviation, $\% \mathrm{CV}=\%$ coefficient of variation. 
to endurance training. Humerus trabecular bone had increased anisotropic orientation, consistent with loadinduced bone remodeling. Cortical bone showed increased bone and tissue mineral density, with no change in volume, suggesting that changes in bone mass are due to increased tissue mineralization. Importantly, the supraspinatus tendon did not have diminished mechanical properties, indicating that this protocol was not injurious to the tendon.

Although the adaptations to exercise found in this study are mild, they are consistent and present across multiple tissues using multiple assays. This was the first study that sought to develop and validate a beneficial exercise protocol that could be directly compared to the established rat supraspinatus overuse proto$\mathrm{col}^{3}$. The rat overuse model has been used in several experiments and replicates many of the changes seen in human tendinopathy, including alterations in mechanical properties, collagen organization, proteoglycans, cell stress, and matrix degrading enzymes. In this study, we confirm that rats undergoing a mild treadmill protocol can also replicate many of the changes seen in human adaptation to beneficial exercise. As with all animal models, the rat may not fully replicate the human condition; however, it has been shown that the supraspinatus in the rat passes under an enclosed arch and has bony architecture similar to humans, replicating the structure and function of the human supraspinatus ${ }^{18}$. This study is further limited by the quality of tissue available, small sample size, and single time point investigated. Additionally, we focused only on the beneficial exercise protocol compared to normal cage activity and did not make any comparisons to the overuse protocol.

In this study, body weight gain decreased, though not significantly, in exercising animals. Although reduced body weight is commonly seen in animals and humans with endurance training, sometimes this change is not detected ${ }^{17}, 19,20$, often due to increased food intake ${ }^{21}$. In this study, food was provided to the animals ad libitum, and intake was not measured. The response of tendon to non-injurious exercise is debated, with some studies reporting increased elastic mechanical properties and cross-sectional area 22,23 and other studies reporting no change ${ }^{24,25}$. In this experiment, we detected no change in tendon cross-sectional area or elastic modulus; however, we found significantly increased failure load and trends toward increased failure stress and collagen content, suggesting that the supraspinatus tendon is responding beneficially to this exercise protocol. Increased collagen content is consistent with other studies that have reported increased collagen mRNA with exercise ${ }^{22,26}$. In contrast to some studies that have reported trends toward increased bone strength with exercise ${ }^{17,20}$, other studies, including this one, found no changes in humerus mechanics following moderate treadmill training ${ }^{27}$, despite beneficial bone structure and mineralization changes detected by $\mu \mathrm{CT}$. The lack of changes found with 3-point bend testing may be due to the small sample size and relatively high variation in mechanical testing outcomes compared to $\mu \mathrm{CT}$.
We validated that a mild treadmill training protocol induces systemic and local shoulder adaptations consistent with beneficial exercise without injuring the supraspinatus tendon. This is the first study that establishes a non-injurious rat exercise protocol for the shoulder. Future studies can use this new model to compare to the established rat supraspinatus overuse model to differentiate beneficial adaptations and maladaptations to loading over time.

\section{Acknowledgments}

This study was supported by the Pennsylvania Muscle Institute (T32 AR 053461) and the Penn Center for Musculoskeletal Disorders (NIH/NIAMS P30 AR 050950). The authors thank Adam Caro, DVM for assistance in dissections and Allison Altman, PhD for assistance in $\mu \mathrm{CT}$ imaging.

\section{References}

1. van der Windt DA, Koes BW, de Jong BA, Bouter LM. Shoulder disorders in general practice: Incidence, patient characteristics, and management. Ann Rheum Dis. 1995;54(12):959-964.

2. Sein ML, Walton J, Linklater J, et al. Shoulder pain in elite swimmers: Primarily due to swim-volume-induced supraspinatus tendinopathy. Br J Sports Med. 2010;44(2):105-113.

3. Soslowsky LJ, Thomopoulos S, Tun S, et al. Neer award 1999. Overuse activity injures the supraspinatus tendon in an animal model: A histologic and biomechanical study. J Shoulder Elbow Surg. 2000;9(2):79-84.

4. Arya S, Kulig K. Tendinopathy alters mechanical and material properties of the achilles tendon. J Appl Physiol. 2010;108 (3):670-675.

5. Padulo J, Oliva F, Frizziero A, Maffulli N. Muscles, Ligaments and Tendons Journal. Basic principles and recommendations in clinical and field science research. MLTJ. 2013;4:250-252.

6. Sprague DawleyRat [Internet]. Charles River Laboratories; 2014 [cited October 17, 2014]. Available from: http://www.criver.com/products-services/basic-research/find-a-model/ sprague-dawley-rat.

7. Thomopoulos S, Williams GR, Soslowsky LJ. Tendon to bone healing: Differences in biomechanical, structural, and compositional properties due to a range of activity levels. J Biomech Eng. 2003;125(1):106-113.

8. Perry AK, Blickhan R, Biewener AA, Heglund NC, Taylor CR. Preferred speeds in terrestrial vertebrates: Are they equivalent? J Exp Biol. 1988;137:207-219.

9. Sullivan TE, Armstrong RB. Rat locomotory muscle fiber activity during trotting and galloping. J Appl Physiol Respir Environ Exerc Physiol. 1978;44(3):358-363.

10. Committee for the Update of the Guide for the Care and Use of Laboratory Animals. Guide for the Care and use of Laboratory Animals. 8th ed. National Research Council, editor. Washington, D.C. National Academies Press; 2011.

11. Peltz CD, Perry SM, Getz CL, Soslowsky LJ. Mechanical properties of the long-head of the biceps tendon are altered in the presence of rotator cuff tears in a rat model. J Orthop Res. 2009;27(3):416-420.

12. Barton ER, Gimbel JA, Williams GR, Soslowsky LJ. Rat supraspinatus muscle atrophy after tendon detachment. J Orthop Res. 2005;23(2):259-265.

13. Peltz CD, Dourte LM, Kuntz AF, et al. The effect of postopera- 
tive passive motion on rotator cuff healing in a rat model. $J$ Bone Joint Surg Am. 2009;91(10):2421-2429.

14. Dourte LM, Pathmanathan L, Mienaltowski MJ, Jawad AF, Birk DE, Soslowsky LJ. Mechanical, compositional, and structural properties of the mouse patellar tendon with changes in biglycan gene expression. J Orthop Res. 2013;31(9):1430-1437.

15. Neuman RE, Logan MA.The determination of hydroxyproline. J Biol Chem. 1950;184(1):299-306.

16. Bouxsein ML, Boyd SK, Christiansen BA, Guldberg RE, Jepsen KJ, Muller R. Guidelines for assessment of bone microstructure in rodents using micro-computed tomography. $\mathrm{J}$ Bone Miner Res. 2010;25(7):1468-1486.

17. Raab DM, Smith EL, Crenshaw TD, Thomas DP. Bone mechanical properties after exercise training in young and old rats. J Appl Physiol. 1990;68(1):130-134.

18. Soslowsky LJ, Carpenter JE, DeBano CM, Banerji I, Moalli MR. Development and use of an animal model for investigations on rotator cuff disease. J Shoulder Elbow Surg. 1996;5 (5):383-392.

19. Saville PD, Whyte MP. Muscle and bone hypertrophy. positive effect of running exercise in the rat. Clin Orthop Relat Res. 1969;65:81-88.

20. Myburgh KH, Noakes TD, Roodt M, Hough FS. Effect of exercise on the development of osteoporosis in adult rats. J Appl Physiol. 1989;66(1):14-19.

21. Baldwin KM, Fitts RH, Booth FW, Winder WW, Holloszy JO. Depletion of muscle and liver glycogen during exercise.pro- tective effect of training. Pflugers Arch. 1975;354(3):203212.

22. Heinemeier KM, Skovgaard D, Bayer ML, et al. Uphill running improves rat achilles tendon tissue mechanical properties and alters gene expression without inducing pathological changes. J Appl Physiol. (1985). 2012;113(5):827-836.

23. Seynnes OR, Erskine RM, Maganaris CN, et al. Training-induced changes in structural and mechanical properties of the patellar tendon are related to muscle hypertrophy but not to strength gains. J Appl Physiol. 2009;107(2):523-530.

24. Legerlotz K, Schjerling P, Langberg H, Bruggemann GP, Niehoff $A$. The effect of running, strength, and vibration strength training on the mechanical, morphological, and biochemical properties of the achilles tendon in rats. J Appl Physiol. 2007;102(2):564-572.

25. Westh $\mathrm{E}$, Kongsgaard M, Bojsen-Moller J, et al. Effect of habitual exercise on the structural and mechanical properties of human tendon, in vivo, in men and women. Scand J Med Sci Sports. 2008;18(1):23-30.

26. Heinemeier KM, Olesen JL, Haddad F, et al. Expression of collagen and related growth factors in rat tendon and skeletal muscle in response to specific contraction types. J Physiol. 2007;582(Pt 3):1303-1316.

27. Warner SE, Shea JE, Miller SC, Shaw JM. Adaptations in cortical and trabecular bone in response to mechanical loading with and without weight bearing. Calcif Tissue Int. 2006; 79(6):395-403. 